\title{
The embryonic development of great ramshorn Planorbarius corneus under the hypomagnetic field
}

\author{
S. S. Moisa ${ }^{1}$, A. A. Zotin ${ }^{2}$, V. V. Tsetlin ${ }^{1}$ \\ ${ }^{1}$ Laboratory of monitoring of radiation conditions of the environment of cosmic stations crews, State Scientific Center of The Russian \\ Federation, Institution of Biomedical Problems of The Russian Academy of Sciences, Moscow, Russia \\ ${ }^{2}$ Laboratory of evolutional developmental biology, Kol'tsov Institute of Developmental Biology of The Russian Academy of Sciences, \\ Moscow, Russia
}

Email address:

butalana07@list.ru (S. S. Moisa), v_tsetlin@mail.ru (V. V. Tsetlin), aazotin@mail.ru (A. A. Zotin)

\section{To cite this article:}

S. S. Moisa, A. A. Zotin, V. V. Tsetlin. The Embryonic Development of Great Ramshorn Planorbarius Corneus under the Hypomagnetic Field. American Journal of Life Sciences. Special Issue: Space Flight Factors: From Cell to Body. Vol. 3, No. 1-2, 2015, pp. 25-31. doi: 10.11648/j.ajls.s.2015030102.15

\begin{abstract}
The effect of a 100-300-fold attenuated geomagnetic field on the embryonic development of great ramshorn Planorbarius corneus and oxidation-reduction properties in water environment were studied in a hypomagnetic chamber. Mainly the hypomagnetic field effected beneficial influence on the development of mollusks: teratogenic effects were less massive, i.e. embryos that first occurred in hypomagnetic conditions were characterized by low death rate. The mobility index increased in embryos on the stage of late veliger and post-metamorphosis. Under the sharp increasing of the magnetic field to the normal level the embryos and juvenile mollusks rapidly perished (practically, their growth was stopped). Type of induction was dependent on adaptation of juvenile P. corneus to a magnetic field. Mollusks growth in the normal geomagnetic field would prefer the conditions with maximal induction, whereas mollusks developed in the hypomagnetic chamber, on the contrary, chose the conditions with minimal induction. It was revealed that the oxidation-reduction potential of water increased as magnetic induction attenuated pointing to a natural decline that testifies about the regular decreasing of internal energy of water molecules, which, in our opinion, is caused the inhibition of the mollusk embryonic development.
\end{abstract}

Keywords: Great Ramshorn Planorbarius Corneus, Embryogenesis, Mobility Index, Oxidation-Reduction Potential of Water, Hypomagnetic Field

\section{Introduction}

All living organisms are the products of long biological evolution as a result some mechanisms, supplying their maximal adaptation to specific conditions of Earth, are formed. Some of environment parameters, such as, for example, gravity, remain more or less constant during significant periods of time, others constantly change. One of the constantly effecting factors is geomagnetic field of Earth. And, although geomagnetic field doesn't stay constant both to induction and to configuration [1], its changing for the period of animal's life can consider negligible. That is sharp changing of geomagnetic field isn't factor of natural selection. Animal reaction on it isn't fast genetically and can be the most diverse.

Effect of changing of natural geomagnetic field of Earth on organisms summing up in monographs [2-4]. There are significantly less works are devoted to the influence of sharp jumps of the induction of geomagnetic field on animals and plants. Although, similar investigations have a special currency in the present in connection with the realization of projects on cosmic flights out of near-earth space [5].

Besides, the question about the ratio of the influences on organism direct effect of hypomagnetic field (HMF) and influence of changing environment due to this factor, is not clear [6]. For the deep understanding of mechanism of indirect effect of HMF on living organisms one should to pay attention to the investigations of physical properties of inner and outer water medium, undergone to weakening geomagnetic field effect. Earlier in model experiments [7, 8] changing of oxidation-reduction properties of water under the effects of environment factors ("electromagnetic" background, ionized radiation, HMF, under eruptions of volcano, solar eclipse). Proceed from these ideas, it is logically to investigate, how change water properties under 
the effect of weakening geomagnetic field. In this respect a special interest is the studying of such water property as oxidation-reduction potential (ORP). ORP should consider a homeostatic characteristic of biological liquids of organism [9].

This work is devoted to the investigation of the influence of weakening induction of geomagnetic field on the development of freshwater gasteropods mollusk Great Ramshorn Planorbarius corneus.

\section{Methods}

Changing of magnetic field induction achieved with the help of hypomagnetic chamber is presented open from one side pipe with $30 \mathrm{~cm}$ in diameter and length $1 \mathrm{~m}$, covered with permaloi inside, which isolated magnetic field. Induction of magnetic field inside of chamber changed at a depth of 0.6 to $30 \mu \mathrm{T}$.

The alterations of water medium properties in chamber were determined by the ORP value of high cleaning water in the separate glass vessel, accommodated into the chamber on the different depth of changing induction of magnetic field with a help of ionomer I-160MI.

Parental individuals $P$. corneus with mass approximately $500 \mathrm{mg}$, grown in aquarium conditions under natural geomagnetic field, kept or in hypomagnetic chamber (10 individuals) or in normal conditions (12 individuals). The conditions of keeping: room temperature (approximately $23^{0} \mathrm{C}$ ); settling not less 2 days water supply $1 l$ in volume; constant feeding with fresh leaves of dandelion; replacement of water and rest of food occurred 2 times a week. Mollusks transferred from hypomagnetic chamber in normal conditions in a 1.5 month. Fertility (the amount of clutches per one individual in twenty-four hours) accounted every week. Clutches and hatching from it mollusks were used for the fulfilling the experiments. All experiments carried out under room temperature (approximately $23^{\circ} \mathrm{C}$ ).

\subsection{Effect of Magnetic Field Changing on Embryonic Development}

1. Clutches, receiving in normal conditions, on the stage of zygote were cut in two and put in $5 \mathrm{ml}$ of settling water supply in plastic Petri caps closed with lid. One of these clutches was grown in the conditions of HMF, another one in normal conditions. Photography of developing clutches realized in interval of 5 min till the hatching of vital embryos with help of microscope AM-311 Dino-Lite Digital Microscope.

The stage of development, embryo line sizes and their age in units of physical time $(\mathrm{t})$ and biological time $(\mathrm{T})$ were determined on getting pictures $[10,11]$.

It should to note that the conditions of experiment fulfilling can't determine the length of way, passing by embryos, and, so, the speed of their motion. Embryo mobility assessed due to the comparison of consequent frame of photography. It is considered that embryo transferred if its position for 5 minutes changed more than 20 minutes. Index mobility is accounted: the part of intervals in which embryo displacement was observed, in \% from total number of 5minute intervals. In 4 clutches 62 embryos were measured.

2. Clutches, receiving in normal conditions, held in these conditions to the beginning of cell division. When blastomeres diverged on not large distance, the part of clutches put in hypomagnetic conditions (HMC). The rest clutches served as control. HMF effect was assessed on two parameters: teratogenesis and the duration of embryogenesis. 34 clutches were studied. Total number of embryo compiled 407 copies.

3. Clutches, hatching in hypomagnetic chamber, transferred in normal conditions on the different stages of development. Embryo vitality was assessed. 12 clutches were investigated. Total number of embryo compiled 106 copies.

\subsection{Effect of Magnetic Field Changing on Juvenile Mollusks}

Juvenile mollusks, hatching from the clutches, laying and staying during all embryonic development in the conditions of HMF, were used. Mollusks after hatching kept in hypomagnetic chamber one by one in $50 \mathrm{ml}$ of settling water supply under the temperature about $23^{\circ} \mathrm{C}$ and constant feeding with fresh leaves of dandelion till age of 14 weeks. Then mollusks extracted from hypomagnetic chamber and their cultivation was lasted under normal geomagnetic field.

Beginning from 10-th week of post-larval development, mollusk mass was measured with the interval 1 time for 1-2 weeks. Weighing was fulfilled in conditions of normal geomagnetic field during 5-10 minutes. Exactness of weighing is $1 \mathrm{mg}$.

\subsection{Survival Potential and fertility of Puberty P. Corneus under the Displacement in Hypomagnetic Conditions and Back}

Puberty mollusks in the amount of 7 copies were put in hypomagnetic chamber for 2 months, after that they were transferred back in normal conditions and were studied yet 2 months. Control animals (7 copies) all time were in normal conditions.

Animals of each group kept together in $0.5 l$ of settling water supply under the constant feeding with leaves of dandelion. Their survival potential and fertility were assessed (the number of clutches per 1 individual in twenty-four hours) 1 time in week.

\subsection{Choosing by Juvenile P. Corneus Induction of Magnetic Field}

For the experiments 2 groups of mollusks, differed in the conditions, in which the development from the moment of zygote clutching and to the moment of experiment: 1-st group - normal conditions (72 individuals in age of 2-3 months); 2-nd group - the conditions of hypomagnetic chamber (31 individuals in age of 1-2 months) were used.

As tank was used the tray size $52 \times 13 \mathrm{~cm}$, filling with settling water supply on the depth $1.5 \mathrm{~cm}$. The tray was 
divided in 10 conditional areas each with length $5.2 \mathrm{~cm}$ and put in hypomagnetic chamber such way that its front edge was able out off chamber on the distance $5.2 \mathrm{~cm}$ from chamber edge. Mollusks of the 1-st group put in the area with minimal magnetic induction, the most distant area from the front edge of the tray $(46,8 \pm 52 \mathrm{~cm})$; mollusks of the 2-nd group - in the area, which was out of the chamber $(0-5.2$ $\mathrm{cm})$. Experiments with mollusks of the different groups carried out in turn during 15 days. In the intervals between the experiments animals kept in usual for group conditions under the constant feeding.

For the effect assessment of the degree of illumination of the different areas of the tray in the separate experiments, the tray was covered with non-transparent lid. Usually food didn't add in the tray. However in one of the experiments with mollusks of the 1-st group in the distant edge of the tray the food (leaves of dandelion) was put. In $24 \mathrm{~h}$ after the beginning of the experiment the amount of animals in each area of the tray, was accounted.

\section{Results and Discussion}

\subsection{Teratogenic Effects}

Embryos in intact clutch of eggs developed normally, independent from that whether the clutches were put in hypomagnetic chamber on the stage of zygote, 2 blastomeres or were hatched in HMC. Majority of embryos hatched in usual periods. Only 2 embryos perished: 1 - on the stage of veliconch and 1 on the stage of crawling embryo. So, $0.5 \%$ embryos died. It ought to note that this value significantly lower than the part of embryos dying in standard conditions of development. Thus, HMC is more beneficial for embryonic development of great ramshorn than the conditions of normal geomagnetic field.

In injuring (cutting in two) clutches the part of teratogenic effects increased. It compiled $18.8 \%(\mathrm{n}=32)$ for embryos, developed in hypomagnetic chamber, and $36.7 \%(n=300)$ for embryos in control. Mainly, the teratogenic effects are connected with the death of embryos on the stage of late gastrula (14 individuals) or veliconch (2 individuals).

The forming of identical twins in HMC was marked in 1 case: blastomeres, created after the 1-st cell division, they fully separated and began to divide every one independently. On the stage of late morula their development stopped (Fig. 1).Similar effect never earlier observed both in normal conditions and in conditions of any experimental effects.

Under the displacement of clutches out of hypomagnetic chamber in normal conditions all embryos died during the day independently on their stage of development: trochophore -8 individuals ( 1 clutch), veliger -8 individuals ( 1 clutch), veliconch -23 individuals ( 3 clutches), crawling embryo -27 individuals ( 3 clutches), hatching embryo -40 individuals (4 clutches).

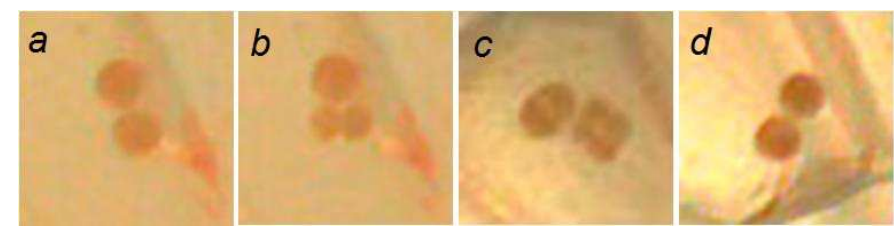

Figure 1. The forming of identical twins $P$. corneus in hypomagnetic chamber. Age of development: $a$ - 0 hours (stage of 2 blastomeres; point); $b$ -2 hours; $c-3$ hours; $d-26$ hours (stage of morula).

\subsection{Effect of GMF on the Period of Development, Sizes and Mobility of Embryos}

The periods of development and sizes of embryos on the different stages in normal conditions and GMC are represented in Table 1 . It is revealed that there are no differences for these parameters.

Table 1. The growth and development of embryo P. corneus.

\begin{tabular}{|c|c|c|c|c|c|c|}
\hline \multirow{3}{*}{ The stage of development } & \multicolumn{3}{|c|}{ Weakening magnetic field $(n=12)$} & \multicolumn{3}{|c|}{ Natural magnetic field $(n=12)$} \\
\hline & \multicolumn{2}{|l|}{ Age } & \multirow{2}{*}{$\begin{array}{l}\text { Size } \\
\mathrm{L}, \boldsymbol{\mu m} \\
\end{array}$} & \multicolumn{2}{|l|}{ Age } & \multirow{2}{*}{$\begin{array}{l}\text { Size } \\
\mathrm{L}, \boldsymbol{\mu m} \\
\end{array}$} \\
\hline & t, Df & $\mathbf{t}, \mathbf{h}$ & & t, Df & $\mathbf{t}, \mathbf{h}$ & \\
\hline Zygote & -1 & $-1,4$ & $107 \pm 2$ & -1 & $-1,5$ & $105 \pm 2$ \\
\hline 2 blastomeres & 0 & 0 & $165 \pm 3$ & 0 & 0 & $161 \pm 3$ \\
\hline 4 blastomeres & 1 & 1,3 & $118 \pm 3$ & 1 & 1,3 & $115 \pm 3$ \\
\hline 8 blastomeres & 4 & 5 & $119 \pm 3$ & 4 & 5 & $121 \pm 3$ \\
\hline Early trochophore & 19 & 25 & $119 \pm 4$ & 19 & 25 & $112 \pm 4$ \\
\hline Middle trochophore & 36 & 32 & $107 \pm 2$ & 35 & 33 & $105 \pm 2$ \\
\hline Late trochophore & 63 & 56 & $193 \pm 4$ & 63 & 56 & $190 \pm 5$ \\
\hline Veliger & 89 & 81 & $266 \pm 14$ & 89 & 81 & $275 \pm 22$ \\
\hline Veliconch & 116 & 105 & $372 \pm 8$ & 118 & 107 & $386 \pm 11$ \\
\hline Crawling embryo & 143 & 129 & $553 \pm 15$ & 143 & 129 & $567 \pm 21$ \\
\hline First hatching & 177 & 160 & $800 \pm 30$ & 175 & 167 & $790 \pm 35$ \\
\hline Last hatching & 200 & 180 & $800 \pm 30$ & 195 & 174 & $790 \pm 35$ \\
\hline
\end{tabular}

Note: took into account only embryos reaching the stage of hatching; $\mathrm{T}$ - biological time (Detlaf); t- physical time (the point of the forming of first fissure of cell division); $\mathrm{L}$ - average maximal size of embryo \pm average mistake; $\mathrm{n}$ - the amount of embryos. 
The results of the determination of embryo mobility are represented in Fig. 2. In those cases, when the mobility is supplied due to the work of eye-lash epithelium of prototrok (trochophore) or sail (veliger), the reliable differences of index mobility in normal conditions and HMC were not discovered.

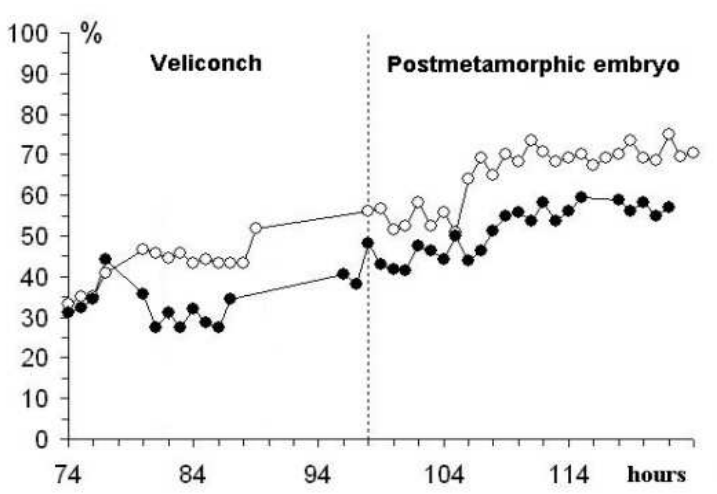

Figure 2. Kinetics of embryo mobility index changing in late embryogenesis. $X$-axis - embryo age; Y-axis - mobility index, accounting for $1 h$ of development. White circles - HMC; black circles - normal conditions. Dotted line - the board between the stages of development.

After embryo settling on capsule wall on the stage of middle veliconch the reliable differenced appeared. It occurred due to the increasing of mobility index in HMC, while in normal conditions index mobility didn't change.

Evaluated degree of mobility in HMC observed also on the stage of post-metamorphosis in comparison with control.

More detail consideration shows that the effect of elevating mobility in HMC observed during all period of development, beginning from the stage of middle veliconch. And average value of index mobility remained approximately constant during the stage of middle veliconch both in experiment and in control. Index mobility increased constantly, beginning from the stage of late veliconch till mollusk hatching in both cases.

The reasons of observed differences of mobility are not clear. One can suppose that it is connected with the peculiarities of embryo motion activity. Veliconch mobility, unlike previous stages, is connected with spasmodic displacement of embryo from one place on capsule surface to another. Embryo first comes off from capsule wall and then again attaches to the capsule in other place. As during metamorphosis and forming of muscle sole embryo passes from the displacement "jumps" to crawling. As hypothesis one can suppose that in HMC occurs the weakening of embryo sole coupling with capsule surface as a result veliconch more often comes off from capsule wall but motion energy of crawling embryo increases. The final decision of this question requires the further investigations.

\subsection{The Growth and Vitality of Juvenile P. Corneus in HMC and under the Displacement in Normal Conditions}

All mollusks grown in HMC in standard curved line of growth which not differed from growth curve under $20-25^{\circ} \mathrm{C}$ in normal conditions (according to earlier finding) (Fig. 3). The displacement of animals out of hypomagnetic chamber and back to weigh them didn't effect significantly on their growth.

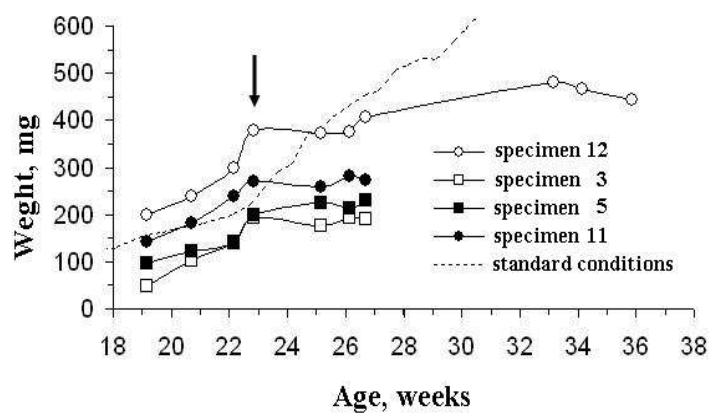

Figure 3. The growth of certain individuals $P$. corneus in $H M C$ with the further their displacement in normal geomagnetic field. Y-axis - body mass of mollusks; X-axis - age after hatching. № individual look at the table 3. Arrow - the moment of mollusk displacement out of hypomagnetic conditions to normal one. Dotted line - the growth in normal conditions.

After the displacement of mollusks in normal conditions the growth speed sharp decreased practically to complete stop growth in all individuals. A majority of mollusks was lost. To the age of 19 weeks ( 5 weeks after the displacement in normal conditions) 4 individuals from 12 (33\%) were alive, and to the age of 27 weeks only 1 mollusk lived out. Survived mollusk lived 62 weeks, its mass after the displacement in normal conditions average remained unchanged and compiled $385 \pm 6 \mathrm{mg}$ ( 7 measurements). For the comparison: according to our data for the maintenance of this mollusk average life duration constitutes $97 \pm 4$ weeks, and average mass reached $1200 \pm 70 \mathrm{mg}$ to the death moment.

\subsection{Survival Potential and Fertility of Puberty P. Corneus under the Displacement in Hypomagnetic Conditions and Back}

All puberty mollusks remained alive during the whole period of investigation both in experimental and in control conditions.

In fertility isn't observed reliable differences too. So, during the period in hypomagnetic chamber it compiled 0.48 \pm 0.21 clutches per individual in twenty-four hours; after the displacement of animals out of HMC in normal ones $-0.65 \pm$ 0.11 clutches per individual in twenty-four hours; under normal conditions $-0.54 \pm 0.17$ clutches per individual in twenty-four hours.

\subsection{Choosing by Juvenile P. Corneus Induction of Magnetic Field}

Majority of mollusks grown in normal conditions during twenty-four hours choose the area with maximal magnetic induction which was out of hypomagnetic chamber. The degree of illumination didn't effect on the choice of mollusks. Under the food is available animals preferred to food and 
choosing the area with food more close to the exit from hypomagnetic chamber (Table 2).

Table 2. The area in the tray, accommodated in hypomagnetic chamber, choosing by juvenile P. corneus

\begin{tabular}{|c|c|c|c|c|c|c|c|c|}
\hline \multirow{4}{*}{ Conditions of experiment } & \multicolumn{8}{|c|}{ Mollusk development } \\
\hline & \multicolumn{4}{|c|}{ natural conditions } & \multirow{2}{*}{\multicolumn{4}{|c|}{ HMC }} \\
\hline & \multicolumn{4}{|c|}{ № experiment } & & & & \\
\hline & 1 & 2 & 3 & 4 & 1 & 2 & 3 & 4 \\
\hline Illumination & No & No & Yes & Yes & Yes & Yes & Yes & Yes \\
\hline Feed is available & No & No & No & Yes & No & No & No & No \\
\hline The amount of mollusk & 72 & 72 & 72 & 72 & 31 & 22 & 17 & 13 \\
\hline Average body mass & 56 & 60 & 85 & 65 & 8 & 15 & 54 & 68 \\
\hline Choosing area*, $\mathrm{cm}$ & \multicolumn{8}{|c|}{ The amount of individuals } \\
\hline $0-5,2$ & 28 & 69 & 42 & 1 & 3 & 1 & 3 & 3 \\
\hline $5,2-10,4$ & 11 & 0 & 11 & 1 & 4 & 0 & 0 & 2 \\
\hline $10,4-15,6$ & 9 & 0 & 3 & 2 & 3 & 0 & 0 & 0 \\
\hline $15,6-20,8$ & 7 & 2 & 4 & 2 & 3 & 2 & 0 & 1 \\
\hline $20,8-26$ & 5 & 0 & 0 & 2 & 0 & 1 & 1 & 0 \\
\hline $26-31,2$ & 3 & 0 & 3 & 1 & 1 & 1 & 0 & 1 \\
\hline $31,2-36,4$ & 2 & 0 & 4 & $37 * *$ & 2 & 4 & 1 & 4 \\
\hline $36,4-41,6$ & 3 & 1 & 0 & 3 & 2 & 1 & 0 & 2 \\
\hline $41,6-46,8$ & 2 & 0 & 1 & $6 * *$ & 0 & 1 & 5 & 2 \\
\hline $46,8-52$ & 2 & 0 & 4 & $17 * *$ & 13 & 11 & 7 & 11 \\
\hline
\end{tabular}

Note: * - the range of length from the tray edge, situated out of the chamber; ** - feed location.

The relative part of mollusks, adapted to normal conditions or HMC, choosing the areas with the different inductions of magnetic field are represented in Fig. 4.

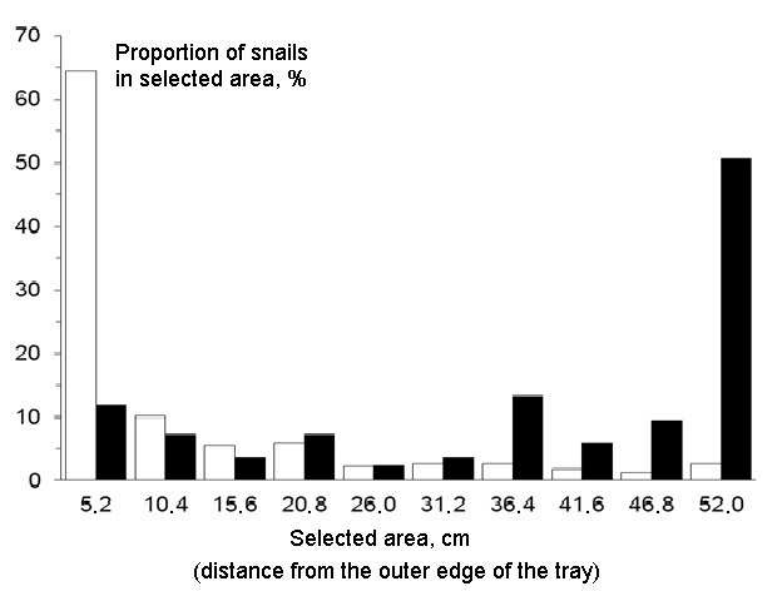

Figure 4. The part of mollusks choosing the different area of the tray. White columns - mollusks, grown in normal conditions; black columns - mollusks, grown in $H M C$.
Mollusks grown in hypomagnetic chamber, on the contrary, during twenty-four hours migrated deep into the chamber. Majority of them choose the most distant area from the exit of hypomagnetic chamber, i.e. the area with minimal induction.

One should to note that in the process of carrying out of experiments (15 day) the death of mollusks of the 1-st group didn't observe, while the amount of mollusks of the 2-nd group decreased approximately to $60 \%$ (look at Table 2).

As to the parameters of the development $P$. corneus in different variants of HMC effect in comparison with normal conditions one can mark that HMC decreases embryo mortality, mobility on the stage of trochophore, veliger and early veliconch. While embryo mobility on the stage of late veliconch and post-metamorphosis increases. Under the displacement out of hypomagnetic chamber the mortality significantly increases and growth speed significantly decreases in juvenile mollusks (Table 3 ).

Table 3. Changing of the parameters of P. corneus development in different variants of HMC in comparison with normal conditions

\begin{tabular}{|c|c|c|c|}
\hline Parameter & Displacement in HMC & Development in HMC & Displacement out of HMC \\
\hline \multicolumn{4}{|l|}{ Embryos } \\
\hline Teratogenesis & without distinctions & decreased & death \\
\hline Period of development & without distinctions & without distinctions & $* *$ \\
\hline Growth speed & without distinctions & $*$ & $* *$ \\
\hline $\begin{array}{l}\text { Mobility on the stage of trochophore, veliger and early } \\
\text { veliconch }\end{array}$ & decreased & $*$ & $* *$ \\
\hline $\begin{array}{l}\text { Mobility on the stage of late veliconch and post- } \\
\text { metamorphosis of embryo }\end{array}$ & increased & $*$ & $* *$ \\
\hline \multicolumn{4}{|l|}{ Juvenile mollusks } \\
\hline Mortality & $*$ & without distinctions & Significantly increased \\
\hline Growth speed & $*$ & without distinctions & Significantly decreased \\
\hline \multicolumn{4}{|l|}{ Puberty mollusks } \\
\hline Mortality & without distinctions & without distinctions & without distinctions \\
\hline Fertility & without distinctions & without distinctions & without distinctions \\
\hline
\end{tabular}

Note: $*$ - the experiments were not carried out; $* *$ - the experiments cannot be done. 


\subsection{Effect of Weakening of Magnetic Field on ORP Value}

It is first revealed that as the weakening of the induction of magnetic field in hypomagnetic chamber the ORP value of water medium increased (Fig. 5), that testifies about naturally determined "decreasing of inner energy of water molecules" [12] and increasing of its oxidative properties.

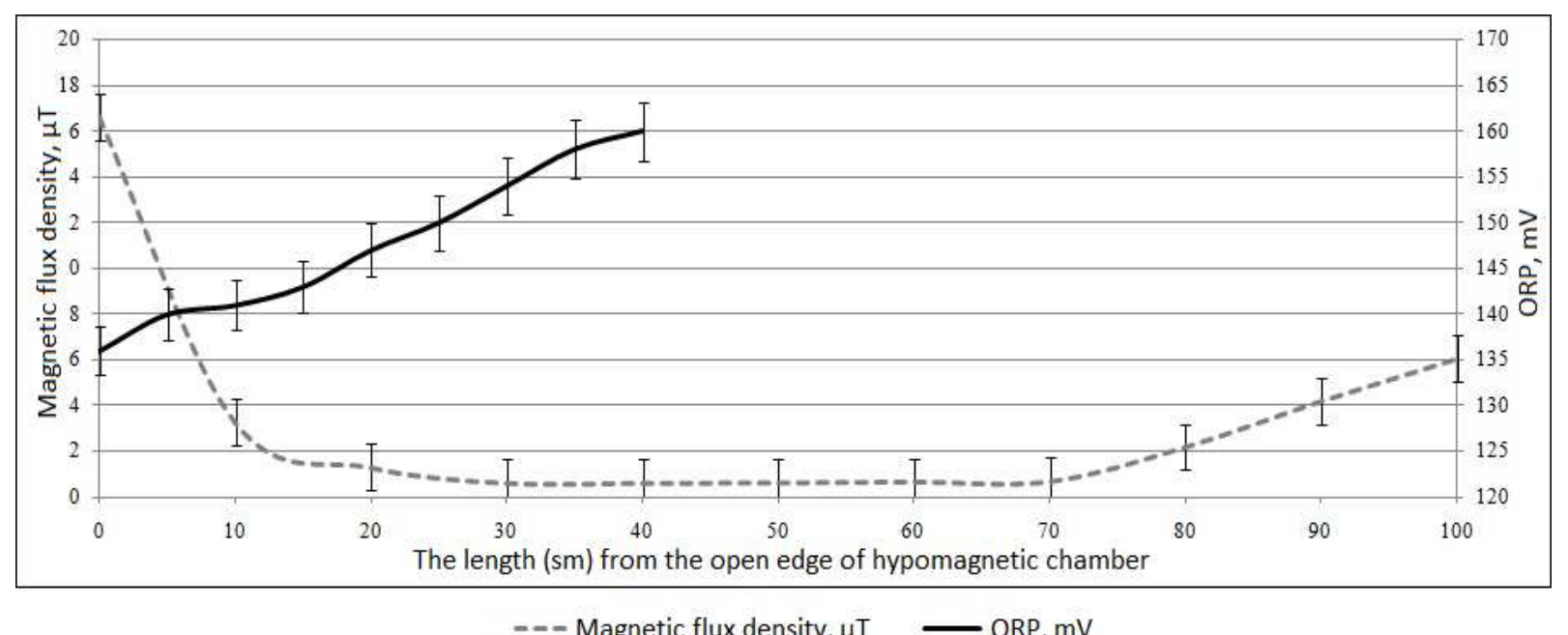

Figure 5. Dependence on ORP and magnetic flux density.

Water, more specifically water basis of living organisms, is the universal receptor of the fields and amplifier of its action. Under a 100-300-fold attenuated geomagnetic field the generation of electrons in water molecules decreases (the work of electron leaving increases). So long as living organisms are water structures the amount of water molecules in excited state which are capable to give back the electrons decreases and ORP value grows and dissolving water properties change under HMF. ORP characterizes the state of inner biological environment of organism. Transport of electrons and protons are also managed by ORP of living mediums of organism. It is established that background (integral) increments of ORP of tissue system structures and liquid biological mediums are varied in the rate of -100 to $400 \mathrm{mV}$ [9] under the using of argentum chloride electrode. The increment of ORP in tissue's mediums by a factor of $+0.01 \mathrm{~V}$ corresponds to 2-3-fold changing in ratio of work for electron transfer from oxidizing compound or element to reducing. Under the decreasing of the amount of excited water molecules due to HMF a less of its amount penetrates into the cells, water medium of cytoplasm, organelles and biochemical functions occurring in it are activated in less degree, i.e. the metabolic processes inhibit, apparently, the slowdown of biochemical processes under a sharp increase of magnetic field to the normal strength caused the revealed stop of growth and death of the majority of juvenile mollusks on experiments. Besides that, from a position of the alteration of water medium state under HMF effect one should to consider the established increasing of embryo mobility index on the stage of middle veliconch and on the stage in post-metamorphosis, i.e. the alteration of water medium state under HMF led to the changing of embryo P.corneus mobility, that testifies about the definite factor of decreasing geomagnetic field effect is the alteration of water medium state. It was shown earlier the decreasing of spontaneous motion activity of eyelash-infusorium spirostoms (Spirostomum ambiguum Ehbg.) - spirostoms, accommodated in the samples of water processing by mixed $\gamma$-neutron irradiation that is the first answer of animals for the transformation of water properties as, it is known, one-cell organisms are a good indicators of subtle alterations in the environment [13].

So long as great ramshorn belongs to trochophor animals it forms a special larva-trochophore, on the apical pole on it is an apical sensor organ - "larval brain", which takes part in catching of outer signals and launching of metamorphosis. These nervous cells effect on the rates of development. As it is known [14], the apical organ of trochophore of great ramshorn consists of 2 neurons. Every one of them has a short appendix coming out and ending with the bundle of sensitive eyelashes, and long appendix going under the eyelash fields. They are on the ventral surface, branch out and form a net of thin filaments with the dilations (varicoses), from which neurons throw out physiological active substances. Exceptionally serotonin synthesizes in great ramshorn (on the stage from trochophore to middle veliger). As it is established [14], under the neuron activation the development of the larvas of great ramshorn slowdowns under serotonin (in 3-4 times), under the decreasing of neuron activation the reliable accelerated development is observed. Proceed from these concepts, one can suppose that weakening magnetic field activates neurons of trochophore $P$. corneus as the result the slowdown of the development till embryo death in our experiment under a sharp increase of the magnetic field to normal level.

Summarizing the finding (table 3 ) it is necessity to note that the decreasing of magnetic field occurs mainly beneficial effect on the development of P.corneus. Embryos which first 
were in GMF, are characterized by decreasing mortality.

However, the alteration of induction in increasing side, occurs serious pathological effect on animals: embryos rapidly death and juvenile mollusks practically stop their growth and majority of them death too. For the mature mollusks the alterations of vitality and fecundity isn't revealed.

\section{Conclusions}

1 Embryos on the stage of late veliger and postmetamorphosis mobility index in GMF increases in comparison with the conditions of normal geomagnetic field. Perhaps, the degree of embryo sole coupling with capsule surface has disturbances under GMF. The check of this hypothesis requires the additional researches.

2 The part of teratogenic effects decreases in GMF.

3 A sharp increase of the magnetic field to natural level leads to embryo death and the majority of juvenile mollusks. The growth of juvenile mollusks practically stops.

4 Type of induction is dependent on adaptation of juvenile P.corneus to a magnetic field. Mollusks, grown in the normal geomagnetic field, prefer the conditions with maximal induction, whereas mollusks developed in the hypomagnetic chamber, on the contrary, prefer the conditions with minimal induction.

5 It is established that the oxidation-reduction potential of water increases as magnetic induction attenuated pointing to a natural decline, it, evidently, caused the inhibition of studying processes of embryonic development of P.corneus.

\section{References}

[1] Koronovsky N.V.,"Magnetic field of geological Past of Earth,"System of life activity support,No.5,1996, pp. 56-63.

[2] Schulten K.,"Magnetic field effects in chemistry and biology," In Festkörperprobleme (J. Treusch, ed.). Braunschweig: Vieweg, vol. 22, 1982, pp. 61-83.

[3] Mizun Yu.G., Mizun P.G., "Space and Health,” M., 1984, $144 p$ (in Russian).
[4] Gould J.L.,"Magnetic Field Sensitivity in Animals," Ann. Rev. Physiol.,vol. 46,1984, pp. 585-598.

[5] Nefedova E.L., Levinskich M.A., Derendyaeva T.A., Tsetlin V.V.,"The investigation of model effects of low doses of ionized radiation under the lowering level of geomagnetic field on growing characteristics of the seeds of high plants conforming to the conditions of long-term interplanetary expeditions," Proceedings of VIII International conference «Space and Biosphere». 28 September - 3 October 2009. Sudak. Ukraine. Kiev. 2009,pp. 226-227(in Russian).

[6] Tsetlin V.V., Zenin S.V., Golovkina T.V. et al.,"The role of water medium in the mechanism of action of supperlow doses of ionized radiation,"Biomeditsinski etehnologii I radioelectronika, No. 12,2003,pp. 20-25 (in Russian).

[7] Tsetlin V.V.,"The investigation of water reaction on the variations of cosmo-physical and geophysical factors of environment,"Aviakosm. I ekol. med.,No. 6,2010, pp. 2631(in Russian).

[8] Tsetlin V.V., Fainshtein G.S.,"About the influence of cosmophysical, geophysical and radiation factors on electro-physical and biological properties of water,"Metafizika, No. 2 (4),2012, pp. 81-89(in Russian).

[9] Bahir I.M.,"The determination of the terms "water" and "solution" conforming to technology of electro-chemical activation,"Elektrohimicheskaya aktivacia v meditsine, selskom hozjaistve, promyshlennosti, No. 14, 1999, pp. 4147(in Russian).

[10] Detlaf T.A., Detlaf A.A.,"Unsized criterions as a method of quantitative characteristic of animal development,"Matematicheskaya biologia razvitia, M. Nauka, 1982,pp. 25-39(in Russian).

[11] Zotin A.A., Kleimenov S.Yu.,"The speed of oxygen consumption in embryo development of Lymnaea stagnalis (Gastropoda),’Ontogenez,vol. 37,No. 3, 2006, pp. 167-172.

[12] RassadkinYu..P.,“Water usual and unusual,” M., 2008, 840 p. (in Russian)

[13] Tsetlin V.V., Zenin S.V., Lebedeva N.E.,"Mechanism of the effect of supperlow doses of ionized radiation on water medium," Biomed. Tehnologii I radioelektronika,No. 6, 2005,pp. 53-58.

[14] Voronezhskaya E.E., Nezlin L.P., Habarova M.Yu.,"What tell snails to your larvas, "Priroda, No. 2,2008, pp. 14-22(in Russian). 